\title{
ANÁLISIS FLORÍSTICO DE LA CAÑADA, QuerÉTARO, MÉXICO
}

\author{
José Alejandro CabreRA-Luna y MARICELA GÓmeZ-SÁnCheZ ${ }^{1}$ \\ Licenciatura en Biología, Facultad de Ciencias Naturales, Universidad Autónoma de Querétaro, \\ Av. de Las Ciencias s/n, C.P. 76230, Juriquilla, Querétaro. \\ ${ }_{1}$ Autor para correspondencia. Correo-e: gomezs@uaq.mx
}

\begin{abstract}
Resumen: La localidad La Cañada, pese a su cercanía con la ciudad de Querétaro, es una área con bosque tropical caducifolio y matorral xerófilo medianamente conservados. Este análisis florístico registra nueve taxa infraespecíficos, 264 especies, 199 géneros y 76 familias. De éstas, Asteraceae y Cactaceae tienen el mayor número de especies. En La Cañada se conserva un alto porcentaje de la flora nativa y se alberga la mayoría de las formas de vida registradas para plantas vasculares. Diez y siete porciento de las especies son endémicas de México y Mammillaria mathildae es endémica del Valle de Querétaro. Se registran cinco especies que están incluidas en la Norma Oficial Mexicana de especies amenazadas. Dos especies del Bajío están amenazadas y dos, previamente colectadas en el área, probablemente son extinciones recientes de Querétaro. La Cañada es la región más rica y diversa del Valle de Querétaro, albergando $11.3 \%$ de las especies del total de la flora estatal, siendo así una zona de alta prioridad para su manejo y conservación.
\end{abstract}

Palabras clave: bosque tropical caducifolio, diversidad, flora, riqueza taxonómica.

Abstract: Despite its vicinity to Querétaro City, the La Cañada locality is a moderately preserved zone with deciduous tropical forest and xeric scrub. In this floristic study, nine infraspecific taxa, 264 species, 199 genera and 76 families were recorded. Among these, Asteraceae and Cactaceae comprised the largest numbers of species. At La Cañada a high percentage of the native flora is preserved, and the majority of biological forms recorded for vascular plants. Seventeen percent of the species are endemic to Mexico and Mammillaria mathildae is endemic to the Valley of Querétaro. Five species are included in the Mexican Official Norm of Endangered Species. Two species from the El Bajío region are threatened and two species, previously collected from the locality, probably represent examples of recent extinctions from Querétaro. La Cañada is the locality with the highest recorded richness and diversity within the Valley of Querétaro, supporting $11.3 \%$ of the species of the state flora. Our results indicate that La Cañada is an important area that requires sound management for its conservation.

Key words: diversity, flora, taxonomic richness, tropical deciduous forest.

$\mathbf{M}$ éxico es uno de los países con mayor riqueza y diversidad de especies vegetales y los intentos por cuantificar con precisión esta riqueza y diversidad han sido cuantiosos. Sin embargo, todavía enfrentamos la falta de un inventario completo y depurado de todas las especies conocidas y un número de plantas no han sido descritas y a menudo ni siquiera descubiertas (Rzedowski, 1991a). A pesar de las floras estatales y regionales que brindan el conocimiento florístico de México, se estima que cerca de $30 \%$ del territorio nacional no ha sido estudiado florísticamente y existen aún áreas insuficientemente exploradas
(Dávila y Sosa, 1994). Un buen estudio florístico es el primer paso para conocer la riqueza de especies vegetales y es la base para generar y establecer estrategias de restauración, manejo y conservación de nuestros recursos vegetales. En este contexto se estudió la riqueza florística de la localidad La Cañada, en los municipios Querétaro y El Marqués, en el estado de Querétaro. La Cañada es una área que carece de estudios de su riqueza y diversidad de especies vegetales y, al igual que otras áreas de México, enfrenta una fuerte amenaza producto de la alteración ecológica producida por diversos agentes. 
Argüelles et al. (1991) citan para el estado de Querétaro 2,334 especies, 918 géneros y 170 familias de plantas vasculares. De esta riqueza estatal, 55 familias, 145 géneros y 204 especies están presentes en el municipio El Marqués, predominando en esta área las familias Asteraceae, Cactaceae, Euphorbiaceae y Fabaceae. Zamudio et al. (1992) señalan para La Cañada la presencia de matorral xerófilo, el cual alterna con algunos manchones de bosque tropical caducifolio.

Ante la amenaza que enfrenta la vegetación de La Cañada y el nulo conocimiento florístico actual, desarrollamos esta investigación con el fin de conocer la riqueza florística, origen, formas de vida, distribución y estado de conservación de las especies vegetales.

\section{Área de estudio}

La Cañada se localiza a $3.5 \mathrm{~km}$ al sureste de la ciudad de Santiago de Querétaro (20³6', 20³8' N y 100¹8', $\left.100^{\circ} 20^{\prime} \mathrm{O}\right)$, entre los municipios Querétaro y El Marqués. Abarca una área total de 26 ha y delimita dos poblados, Hércules y La Cañada (figura 1). Su altitud oscila entre 1,850 y 2,030 m s.n.m. en su punto más alto (Anónimo, 1996, 1997, 2001). El clima de la zona es $\mathrm{BS}_{1} \mathrm{~K}$, es decir, semiseco templado con lluvias entre los meses de mayo a septiembre. La temperatura media anual varía entre $18^{\circ} \mathrm{C} \mathrm{y}$ $19^{\circ} \mathrm{C}$, con una máxima de $22^{\circ} \mathrm{C}$ durante el mes de mayo. Fisiográficamente, La Cañada se ubica en la provincia del Eje Neovolcánico y en la subprovincia de las Llanuras y
Sierras de Querétaro e Hidalgo (Anónimo, 1986). La vegetación predominante muestra asociaciones de matorral xerófilo (matorral crasicaule y matorral espinoso), entremezcladas con algunos manchones residuales de bosque tropical caducifolio (Zamudio et al., 1992). A lo largo del río Querétaro se observan elementos de vegetación acuática y riparia, mientras que en otras zonas la alteración ha provocado el establecimiento de pastizal y vegetación secundarios.

\section{Materiales y métodos}

Durante el período 2002-2004 se hicieron colectas semanales, sistemáticas y selectivas, a través de recorridos en La Cañada. El material vegetal se procesó mediante las técnicas convencionales para cada grupo taxonómico siguiendo los criterios de Lot y Chiang (1986). Para cada planta se consideró la localización, la fecha de colecta, así como las características biológicas y del microambiente. Los especímenes se determinaron con ayuda de bibliografía especializada; las briofitas fueron revisadas por especialistas. La colección principal se depositó en el herbario QMEX y los duplicados se enviarán en breve a otros herbarios nacionales.

Para cada especie se recabó información bibliográfica relativa a su forma biológica, origen, distribución y estado de conservación, misma que se complementó con observaciones de campo. Las categorías de formas de vida se establecieron según los criterios de Rzedowski (1978) con

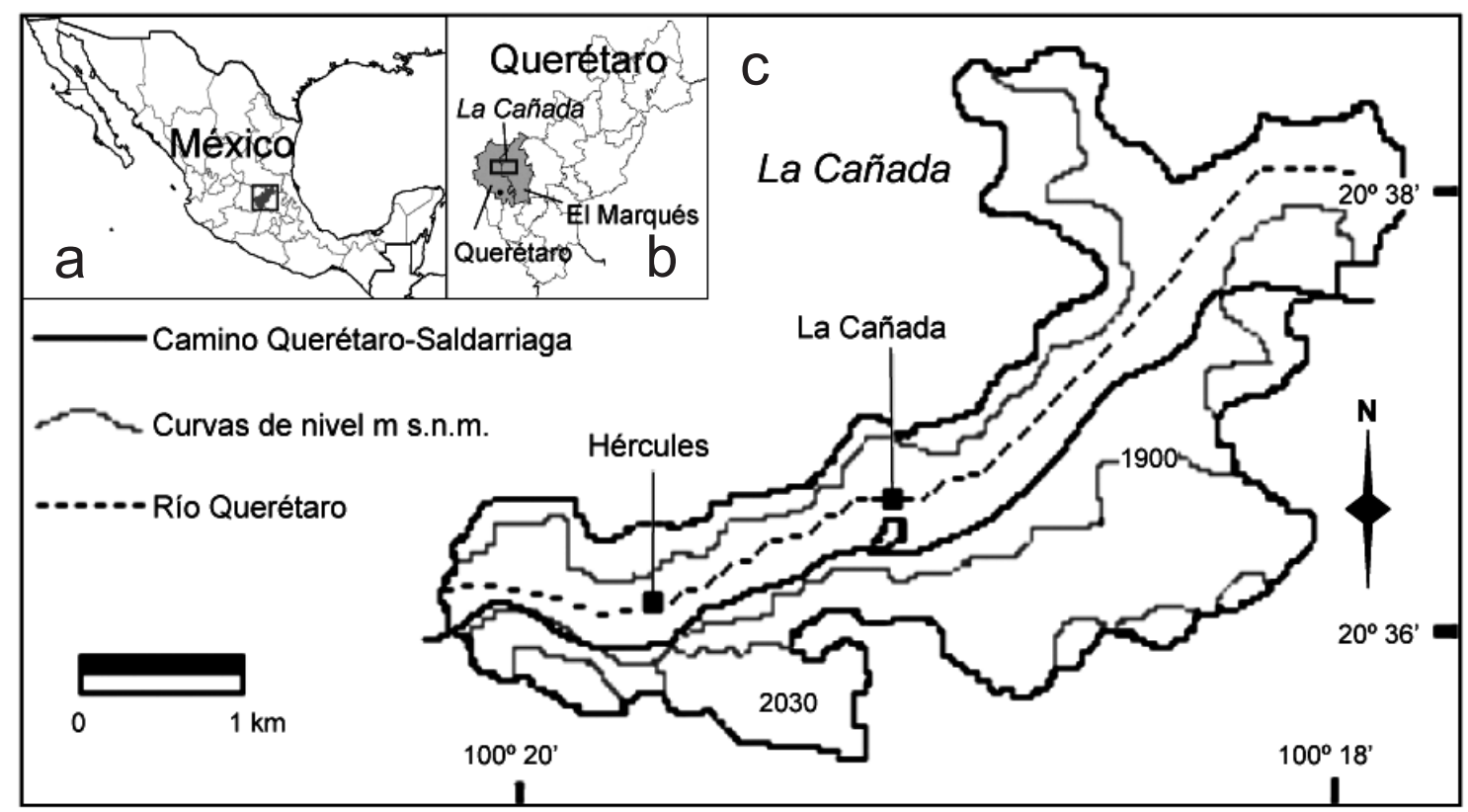

Figura 1. Localización del área de estudio. (a) Ubicación del estado de Querétaro, (b) Municipios de Querétaro y El Marqués, (c) La Cañada. 


\section{ANÁLISIS Florístico de La CAÑADA, QuerÉTARo}

algunas modificaciones. Para determinar el estado de conservación se usó el criterio de la lista roja de la Unión Internacional para la Conservación de la Naturaleza (UICN) (Walter y Gillett, 1998) y la Norma Oficial Mexicana (NOM-059-ECOL-2001; SEMARNAT, 2001) para especies amenazadas y se añadieron las observaciones directas de campo.

Con esta información se generó un catálogo florístico (apéndice 1) donde las familias, los géneros, las especies, subespecies y variedades siguen un orden alfabético. La determinación de algunos especímenes no fue posible. Éstos serán revisados por especialistas, pues probablemente se trata de entidades nuevas o registros nuevos para la flora estatal. Respecto a los grupos taxonómicos, se siguieron los criterios propuestos por Mickel y Beitel (1988) para Pteridophyta y grupos afines, Cronquist (1981) para Magnoliopsida y Dahlgren et al. (1985) para Liliopsida. Los géneros de gramíneas se estandarizaron según Clayton y Renvoize (1986), las plantas acuáticas según Lot et al. (1999) y para la familia Cactaceae se siguió el criterio de Guzmán et al. (2003). La nomenclatura de los géneros y especies se cita de acuerdo con las bases de datos del International Plant Name Index (2004) y la base de datos del Missouri Botanical Garden ( $\mathrm{W}^{3}$ Tropicos,
2005). Los nombres de autores se citaron de acuerdo con Brummit y Powell (1992).

\section{Resultados}

Riqueza taxonómica. La riqueza taxonómica se manifiesta en 76 familias, 199 géneros, 264 especies, cinco variedades y cuatro subespecies. Las Briofitas son el grupo con la menor riqueza seguido de las Pteridofitas. Las Magnoliopsida o dicotiledóneas constituyen el conjunto de plantas vasculares con mayor riqueza y el grupo dominante en La Cañada (cuadro 1).

Cuadro 1. Composición taxonómica de la flora de La Cañada.

\begin{tabular}{lccccc}
\hline Grupo & Familias & Géneros & Especies & Variedades Subespecies \\
\hline Bryophyta & $3(3.9 \%)$ & $6(3 \%)$ & $7(2.7 \%)$ & - & - \\
Pteridophyta & $4(5.3 \%)$ & $4(2 \%)$ & $11(4.2 \%)$ & - & - \\
Magnoliopsida & $58(76.3 \%)$ & $164(82.4 \%)$ & $213(80.6 \%)$ & 5 & 4 \\
Liliopsida & $11(14.5 \%)$ & $25(12.6 \%)$ & $33(12.5 \%)$ & - & - \\
Total & 76 & 199 & 264 & 5 & 4 \\
\hline
\end{tabular}

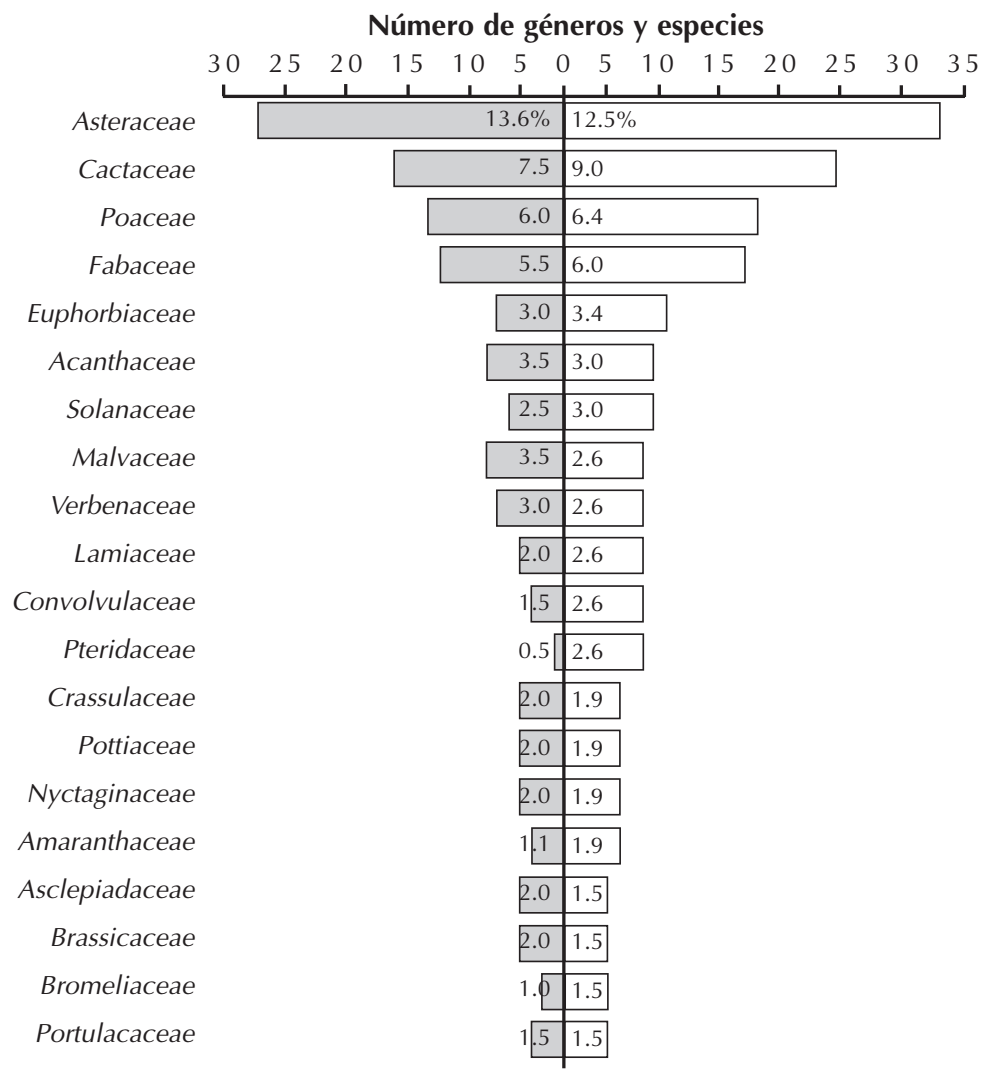

Figura 2. Número y porcentaje relativo de géneros (barras grises) y especies (barras blancas) por familia registradas en La Cañada. 
La familia con mayor número de géneros es Asteraceae con 27 (13.6\%), seguida de Cactaceae con 15 (7.5\%), Poaceae con 12 (6\%) y Fabaceae con 11 $(5.5 \%)$. Las familias más ricas en número de especies son Asteraceae con 33 (12.5\%), Cactaceae con 24 (9\%), Poaceae con 17 (6.4\%), Fabaceae con 16 (6\%) y Euphorbiaceae con 9 (3.4\%) (figura 2). Los géneros con mayor riqueza son Cheilanthes con siete especies (2.6\%), Opuntia con seis (2.3\%), Ipomoea con cinco (1.9\%) y Euphorbia, Solanum y Salvia con cuatro (1.5\%) especies cada uno. El resto de los géneros contiene de una a tres especies (figura 3 ).

Origen de la flora. Del total de especies registradas en La Cañada, $221(83.7 \%)$ son nativas y 43 (16.3\%) son introducidas (cuadro 2). Estas últimas se distribuyen, según su origen, como sigue:

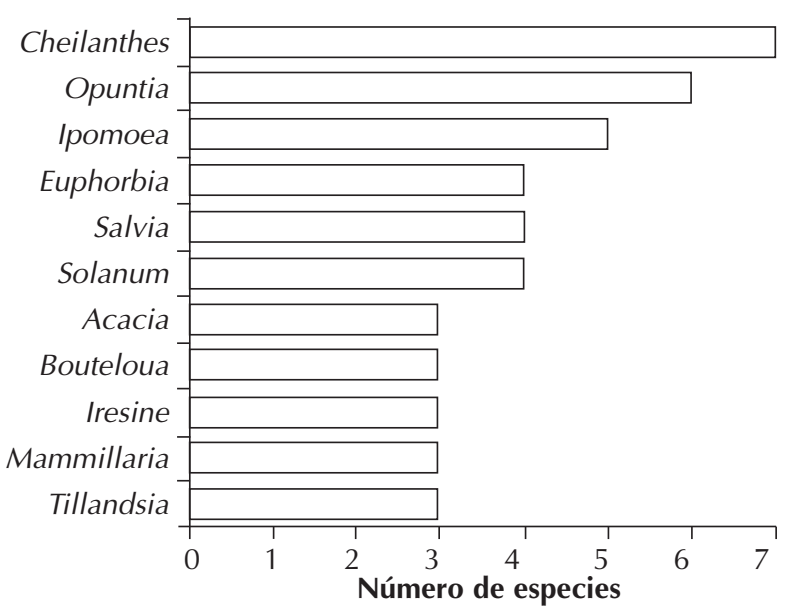

Figura 3. Géneros con mayor número de especies de la flora de La Cañada.

Norteamérica:

Aster subulatus

Cucurbita foetidissima

Lepidium virginicum

Sudamérica:

Aristida divaricata

Schinus molle

Oxybaphus glabrifolius

Zinnia peruviana

\section{Bouteloua hirsuta \\ Euphorbia dentata \\ Oxalis decaphylla}

Baccharis salicifolia

Malvastrum coromandelianum

Petunia parviflora
Cyclanthera dissecta

Helianthus aff. laciniatus

Proboscidea louisiana

Herissantia crispa

Nicotiana glauca

Solanum elaeagnifolium

América tropical (regiones bajas y calientes de América):

Apium leptophyllum

Bidens ferulifolia

Euphorbia hirta var. procumbens

Mollugo verticillata

Euphorbia prostrata

Nopalea cochenillifera

Bouchea prismatica

Ipomoea purpurea

Panicum fasciculatum

Sphaeralcea angustifolia

Euroasiáticas:

Eruca sativa

Portulaca oleraceae

Malva parviflora

Rumex crispus

Plantago major

África:

Aloe vera

Eleusine multiflora

Ricinus communis

Melinis repens

Asia:

Commelina difusa

Gnaphalium luteo-album

Caribe:

Bidens odorata

Europa:

Eragrostis cilianensis

Mediterráneo: Dipsacus sp. (es probable que esta especie se trate de un nuevo registro para la flora mexicana) 
Cuadro 2. Origen de la flora de La Cañada, Querétaro.

\begin{tabular}{lcc}
\hline Origen & Número de especies & Porcentaje \\
\hline México & 221 & 83.7 \\
Sudamérica & 10 & 3.8 \\
América Tropical & 10 & 3.8 \\
Norteamérica & 9 & 3.4 \\
Euroasiática & 5 & 1.8 \\
África & 4 & 1.5 \\
Asia & 2 & 0.8 \\
Caribe & 1 & 0.4 \\
Europa & 1 & 0.4 \\
Mediterráneo & 1 & 0.4 \\
Total & 264 & 100 \\
\hline
\end{tabular}

Formas de vida. La flora de La Cañada concentra la mayoría de las formas de vida registradas para plantas vasculares, enlistándose un total de 14 formas biológicas: árboles, arbustos, bulbosas, crasicaules, epífitas, hierbas anuales, hierbas perennes, hierbas rastreras, hierbas trepadoras o enredaderas, enraizadas emergentes, libre flotadoras, parásitas, rizomatosas y rosetiformes. Las hierbas anuales representan la forma biológica predominante con 60 (22.7\%) especies, seguidas por las hierbas perennes con 59 (22.4\%), los arbustos con 39 (14.8\%) y las crasicaules con $30(11.4 \%)$ especies, respectivamente (cuadro 3 ). Las formas rastrera, trepadora, arbórea y rizomatosa también tienen una incidencia importante.

Distribución y endemismo. 46.6\% (123) de las especies registradas se distribuye ampliamente en América; le siguen con $17.4 \%$ (46) las endémicas de México, $15.9 \%$ (42) se distribuyen en el sur de Estados Unidos y México (Megaméxico 1) y 9.1\% (24) son cosmopolitas (cuadro 4). De las especies endémicas de México, Mammillaria mathildae Kräehenb. et Krainz representa un microendemismo de los alrededores de la ciudad de Querétaro. Actualmente sólo se conocen dos poblaciones de esta especie, la localidad tipo que pertenece a La Cañada, municipio El Marqués (Bravo-Hollis y Sánchez-Mejorada, 1991; Glass, 1998) y la segunda en Los Cajones, municipio de Querétaro, población recientemente descubierta por Hernández y Sánchez (2002). Del total de las especies que crecen en La Cañada y que son endémicas de México, $45.6 \%$ (21) pertenecen a la familia Cactaceae (cuadro 5).

Especies importantes para su conservación. Mammillaria mathildae es una especie catalogada como vulnerable por la UICN y en peligro de extinción por la NOM-059-ECOL2001. El hábitat de esta especie se reduce a poco más de 1 ha
Cuadro 3. Formas de vida presentes en la flora de La Cañada.

\begin{tabular}{lcc}
\hline Forma de vida & Número de especies & Porcentaje \\
\hline Hierbas anuales & 60 & 22.7 \\
Hierbas perennes & 59 & 22.4 \\
Arbustos & 39 & 14.8 \\
Crasicaules & 30 & 11.4 \\
Rastreras & 17 & 6.4 \\
Trepadoras & 16 & 6 \\
Árboles & 14 & 5.3 \\
Rizomatosas & 9 & 3.4 \\
Rosetiformes & 6 & 2.3 \\
Bulbosas & 5 & 1.9 \\
Enraizada emergente & 3 & 1.1 \\
Parásitas & 3 & 1.1 \\
Epífitas & 2 & 0.8 \\
Libre flotadora & 1 & 0.4 \\
Total & 264 & 100 \\
\hline
\end{tabular}

y los agentes de disturbio que afectan considerablemente su población son la ganadería, la extracción de cantera, la contaminación del río Querétaro y la presencia de tiraderos clandestinos de basura y escombro.

Coryphantha radians (DC.) Britton et Rose es una especie que se registra en la lista roja de la UICN y en la NOM-059-ECOL-2001 como rara. Comparte el mismo hábitat de Mammillaria mathildae y su única población consta de cinco individuos, por lo que está sujeta a una alta vulnerabilidad.

Echinocactus platyacanthus Link et Otto está catalogada como vulnerable por la UICN y sujeta a protección especial

Cuadro 4. Distribución de las especies de la flora de La Cañada. Mega México 1 = Sur de Estados Unidos y México, Mega México 2 = México y Centroamérica, y Mega México 3 = Sur de Estados Unidos, México y Centroamérica.

\begin{tabular}{lcc}
\hline Distribución & Número de especies & Porcentaje \\
\hline América & 123 & 46.6 \\
México & 46 & 17.4 \\
Mega México 1 & 42 & 15.9 \\
Cosmopolitas & 24 & 9.1 \\
Mega México 3 & 17 & 6.4 \\
Mega México 2 & 9 & 3.4 \\
Disyunta en América & 3 & 1.2 \\
Total & 264 & 100 \\
\hline
\end{tabular}


José Alejandro CABRERA-LunA y MARICELA GóMEZ-SÁNCHEZ

Cuadro 5. Endemismos registrados en la flora de La Cañada, Querétaro

\begin{tabular}{lcc}
\hline Familia & Número de especies & Porcentaje \\
\hline Cactaceae & 21 & 45.6 \\
Crassulaceae & 4 & 8.7 \\
Asteraceae & 3 & 6.5 \\
Fabaceae & 3 & 6.5 \\
Asclepiadaceae & 2 & 4.3 \\
Amaryllidaceae & 2 & 4.3 \\
Malvaceae & 2 & 4.3 \\
Apocynaceae & 1 & 2.2 \\
Brassicaceae & 1 & 2.2 \\
Cucurbitaceae & 1 & 2.2 \\
Orchidaceae & 1 & 2.2 \\
Poaceae & 1 & 2.2 \\
Rhamnaceae & 1 & 2.2 \\
Sterculiaceae & 1 & 2.2 \\
Verbenaceae & 1 & 2.2 \\
Zygophyllaceae & 1 & 2.2 \\
Total & 46 & 100 \\
\hline
\end{tabular}

por la NOM-059-ECOL-2001. Esta especie crece cerca de casas habitación e integra una población de 10 individuos jóvenes. No obstante, al año siguiente de que las plantas fueron observadas se presentó un incendio y se abrieron nuevas áreas para la construcción. Esto sugiere que, al menos localmente, Echinocactus platyacanthus ha desaparecido o está por desaparecer de La Cañada.

Erythrina coralloides DC. se registra en la NOM-059ECOL-2001 como especie amenazada. No obstante, en La Cañada es una especie común que crece a lo largo del área de estudio y es cultivada por los pobladores de La Cañada y Hércules con fines ornamentales. Así mismo, esta especie es común en distintas partes de México (Espinosa, 1979; Mc Vaugh, 1987), por lo que se sugiere una revaloración de su estado de conservación.

Laelia aff. speciosa (Kunth) Schltr., se registra en la NOM-059-Ecol-2001 como especie amenazada y como vulnerable por la UICN. En La Cañada, esta orquídea habita en la ladera sur y crece sobre mezquite (Prosopis laevigata (Humb. et Bonpl. ex Willd.) M.C.Johnst.); no obstante, es importante señalar que durante este trabajo no fue posible localizarla en fase reproductiva, por lo que su determinación taxonómica se hizo con base en sus rasgos vegetativos.

Bursera palmeri S.Watson y Ceiba aesculifolia (Kunth) Britten et Baker f. son especies frecuentes en el bosque tropical caducifolio de los alrededores de la ciudad de
Querétaro (Zamudio et al., 1992). Sin embargo, en La Cañada ambas especies son escasas y sólo están creciendo en lugares protegidos y húmedos. En la región del Bajío Bursera palmeri y Ceiba aesculifolia se consideran especies amenazadas (Rzedowski y Guevara-Féfer, 1992; Carranza-González y Blanco-García, 2000).

Otras especies que no se registran en la lista de la UICN y en la Norma Oficial Mexicana NOM-059-ECOL-2001, pero que ameritan atención especial en La Cañada, son Ferocactus latispinus (Haw.) Britton et Rose, Mammillaria magnimamma Haw., Mammillaria polythele Mart., Pachyphytum compactum Rose, Stenocactus dichroacanthus (Mart. ex Pfeiff.) A.Berger ex Backeb. et F.M.Kunth y Stenocereus queretaroensis (F.A.C.Weber) Buxb. Aunque sus poblaciones son relativamente numerosas y se encuentran bien representadas a lo largo de La Cañada, la mayoría de los individuos crecen cerca de casas habitación y son objeto de explotación con fines ornamentales y de consumo.

Allowissadula floribunda (Schltdl.) Fryxell y Anoda maculata Fryxell son especies escasas en la región del Bajío y están en peligro de extinción. En el estado de Querétaro sólo se han registrado en La Cañada y al parecer, sus poblaciones son escasas y están restringidas a una pequeña área (Fryxell, 1993). Estos registros son previos a este estudio y provienen del Cerro Colorado, región que actualmente es ocupada por casas habitación. Durante este trabajo no fue posible encontrarlas, por lo que posiblemente podrían tratarse de casos recientes de extinción; sin embargo, son necesarios estudios detallados y búsquedas exhaustivas en los alrededores de La Cañada y otras localidades similares en el estado para descartar dicha posibilidad.

\section{Discusión}

La riqueza de La Cañada es producto de una diversidad de ambientes y microambientes que permiten el desarrollo de especies con condiciones ecológicas especiales. También contribuye a esta riqueza la presencia de matorral xerófilo, comunidad vegetal que es considerada rica en endemismos tanto a nivel genérico como específico (Rzedowski, 1978; Rzedowski, 1991a). A pesar de tener una superficie menor que el resto de los cerros y cañadas del Valle de Querétaro, La Cañada con 264 especies, 199 géneros y 76 familias, es el área con mayor riqueza de los alrededores de la ciudad de Querétaro, superando a los cerros Cimatario y Ermitaño (180 especies, 141 géneros y 62 familias) (BaltazarRamírez et al., 2004) y a las cañadas Los Cajones y La Plata (111 especies, 106 géneros y 41 familias) (BaltazarRamírez, 1998). Con base en el listado florístico de Argüelles et al. (1991), La Cañada alberga $11.3 \%$ de las especies, $21.6 \%$ de los géneros y $44.7 \%$ de las familias de plantas vasculares registradas para el estado de Querétaro. Dos familias, 10 géneros y 14 especies provienen de colec- 
tas previas (ver apéndice 1) a este estudio; sin embargo, durante este trabajo no fueron encontradas, por lo que su colecta no fue posible. Esta ausencia es producto de la alteración a la que La Cañada ha estado expuesta, misma que está provocando una pérdida de la diversidad vegetal.

Asteraceae (27/33), Cactaceae (15/24), Poaceae (12/17) y Fabaceae (11/16) son las cuatro familias con mayor riqueza de géneros y también con mayor riqueza de especies. Estas familias reúnen $32.7 \%$ (65) de los géneros y $34 \%$ (90) de las especies de la flora total de La Cañada. Las familias más ricas en géneros también son las más ricas en especies. Esta correspondencia es similar a otras floras de México y Querétaro. La predominancia de estas familias corresponde a regiones montañosas, áridas y semiáridas de México (Rzedowski, 1978; Rzedowski, 1991a; Villaseñor, 2003).

Entre los géneros más ricos destacan Cheilanthes, Opuntia, Ipomoea, Solanum y Salvia. Éstos son algunos de los que tienen mayor número de especies con una amplia distribución en México (Villaseñor, 2004). En el caso de Ipomoea, Solanum y Euphorbia, además de su alta riqueza y amplia distribución, tienen una gran capacidad para colonizar ambientes alterados. Cheilanthes es el género con mayor riqueza de especies (7), misma que está favorecida por la presencia de numerosos microambientes donde la humedad se incrementa. Este género también es el más rico (78 especies) entre las pteridofitas en México (Villaseñor, 2004) y el mejor representado en los matorrales y pastizales mexicanos (Riba, 1998).

A pesar de los diferentes agentes de disturbio que imperan en La Cañada, esta área tiene un alto porcentaje de flora nativa $(83.7 \%)$. No obstante, ya se observa un número considerable de especies introducidas $(43,16.3 \%)$, de las cuales Aloe vera, Elusine multiflora y Melinis repens son especies agresivas y con alta capacidad de propagación. Estas especies competirán fuertemente con las especies nativas por la obtención de recursos, proceso que podría reducir las poblaciones de estas últimas o incluso desplazarlas si no se atiende este aspecto con oportunidad.

Las hierbas constituyen la forma de vida predominante en La Cañada. Este biotipo es común en regiones áridas, ya que confiere a las plantas resistencia al estrés hídrico y a la escasez de suelo; ambas condiciones prevalecen en el área de estudio. Existe una correspondencia de las formas de vida con los tipos de vegetación. Así, las hierbas, las crasicaules y los arbustos crecen en los matorrales y pastizales, mientras que los árboles y los arbustos lo hacen en el bosque tropical caducifolio y la vegetación riparia.

La alta diversidad de formas de vida registradas en La Cañada parece ser producto de la heterogeneidad ambiental, así como de la coexistencia de dos tipos de vegetación: matorral xerófilo y bosque tropical caducifolio. La riqueza de especies y de formas de vida que se citan aquí coinciden con el hallazgo de Rzedowski (1991a), quien señaló que la flora mexicana, además de ser rica en número de especies, es rica en formas biológicas, principalmente en las regiones áridas y semiáridas.

Rzedowski (1991b) considera que 50\% de las especies vegetales de México son endémicas. Esta consideración se ve incrementada al considerar regiones más naturales como el sur de Estados Unidos y Centroamérica (Megaméxico 3). Para La Cañada, 17.4\% (41) de las especies registradas son endémicas de México y de éstas, una (Mammillaria mathildae) representa un microendemismo de los alrededores de la ciudad de Querétaro. Las especies endémicas de México y que crecen en La Cañada son principalmente cactáceas, familia en que se registra un alto endemismo en géneros y especies en la flora mexicana. A su vez, el matorral xerófilo es una de las comunidades vegetales en donde se han registrado los mayores números de especies endémicas de México (Rzedowski, 1991b). Estos dos factores, así como la historia geológica y evolutiva del Eje Neovolcánico, son los causantes principales de que en La Cañada se encuentre un considerable número de endemismos y un caso de microendemismo.

Entre los principales factores que afectan la supervivencia de las plantas en La Cañada está el cambio de uso de suelo, principalmente con fines urbanos, la extracción de cantera y la extracción de plantas con fines ornamentales y de frutos para consumo humano. Es urgente emprender estudios acerca de la dinámica y la estructura de las poblaciones, y la biología reproductiva de Mammillaria mathildae, especie microendémica y en peligro de extinción, para generar programas de propagación, manejo y conservación. Otras especies que también ameritan atención especial son Coryphantha radians, Bursera palmeri, Ceiba aesculifolia, Echinocactus platyacanthus, Ferocactus latispinus, Laelia aff. speciosa, Mammillaria magnimamma y Stenocereus queretaroensis. Allowissadula floribuna y Anoda maculata ameritan estudios de distribución y estatus de conservación en la región del Bajío y en el estado de Querétaro que aclaren su aparente extinción.

Además de contribuir con una riqueza considerable de especies, La Cañada brinda también algunos servicios ambientales como la recarga de acuíferos, posiblemente la reducción de contaminación por $\mathrm{CO}_{2}$ y la existencia de áreas de esparcimiento para la población. Estos valores ameritan que se elabore un plan específico para su conservación y restauración en áreas que así lo requieran (Hobbs y Norton, 1996; Dobson et al., 1997; Palmer et al., 1997; Holmes y Richardson, 1999). De seguir el deterioro en la vegetación y la apertura de áreas para la construcción, así como la falta de un programa que integre aspectos de conservación, uso y manejo de los recursos vegetales, se perderá la riqueza florística, de especies nativas y de formas de vida. De igual forma, la población de Mammillaria mathildae podría desaparecer en corto tiempo. Esto repercutirá primero en la pérdida de diversidad vegetal en los 
José Alejandro CABrera-Luna y Maricela GómeZ-SÁnchez

alrededores de la ciudad de Querétaro y en un segundo plano, pero no menos importante, en la pérdida de diversidad de México.

\section{Agradecimientos}

Agradecemos a Patricia Herrera Paniagua la determinación de las briofitas y al personal del herbario QMEX por facilitar la consulta de material. Robert Jones amablemente revisó el resumen en inglés. Jorge A. Meave, Arturo Solís Magallanes y un revisor anónimo enriquecieron este trabajo con sus valiosas sugerencias. Esta investigación se desarrolló con un apoyo brindado al primer autor dentro del programa PIFI 2003-23-08 y un apoyo parcial a través del proyecto U031, financiado por la Comisión Nacional para el Conocimiento y Uso de la Biodiversidad.

\section{Literatura citada}

Anónimo. 1986. Síntesis Geográfica, Nomenclátor y Anexo Cartográfico del Estado de Querétaro. Instituto Nacional de Estadística, Geografía e Informática, Aguascalientes.

Anónimo. 1996. Carta Topográfica 1:5,000. Ajuchitlán. Instituto Nacional de Estadística, Geografía e Informática, Aguascalientes.

Anónimo. 1997. Cuaderno Estadístico Municipal, El Marqués, Estado de Querétaro. Instituto Nacional de Estadística, Geografía e Informática, Aguascalientes.

Anónimo. 2001. Carta Topográfica 1:5,000. Querétaro. Instituto Nacional de Estadística, Geografía e Informática, Aguascalientes.

Argüelles E., Fernández R. y Zamudio S. 1991. Listado Florístico Preliminar del Estado de Querétaro. Flora del Bajío y de Regiones Adyacentes. Fascículo Complementario II. Instituto de Ecología, A.C., Pátzcuaro.

Baltazar-Ramírez J.O. 1998. Claves de identificación por corteza de árboles y arbustos en bosque tropical caducifolio. Tesis de Licenciatura, Facultad de Ciencias Naturales, Universidad Autónoma de Querétaro, Querétaro. 114 pp.

Baltazar-Ramírez J.O., Martínez y Díaz M. y HernándezSandoval L. 2004. Guía de Plantas Comunes del Parque Nacional "El Cimatario" y sus Alrededores. Universidad Autónoma de Querétaro, Querétaro.

Bravo-Hollis H. y Sánchez-Mejorada H. 1991. Las Cactáceas de México. Vol. 3. Universidad Nacional Autónoma de México, México, D.F.

Brummitt R.K. y Powell C.E. Eds. 1992. Author of Plant Names. Royal Botanical Gardens, Kew.

Carranza-González E. y Blanco-García A. 2000. Familia Bombacaceae. En: Flora del Bajío y de Regiones Adyacentes. Fascículo 90. Instituto de Ecología, A.C., Pátzcuaro.

Clayton W.A. y Renvoize S.A. 1986. Genera Graminum. Grasses of the World. Kew Bulletin. Add. Ser. XIII, Londres.

Cronquist A. 1981. An Integrated System of Classification of Flowering Plants. Columbia University Press, Nueva York.

Dahlgren R.M.T., Clifford H.T. y Yeo P.F. 1985. The Families of Monocotyledons. Springer-Verlag, Nueva York.

Dávila P. y Sosa V. 1994. El conocimiento florístico de México.
Boletín de la Sociedad Botánica de México 55:21-27.

Dobson A.P., Bradshaw A.D. y Baker A.J.M. 1997. Hopes for the future: restoration ecology and conservation biology. Science 277:515-522.

Espinosa G.J. 1979. Leguminosae. En: Calderón de Rzedowski G. y Rzedowski J. Eds. Flora Fanerogámica del Valle de México, pp. 279-354, CECSA, México, D.F.

Fryxell P.A. 1993. Familia Malvaceae. Flora del Bajío y de Regiones Adyacentes. Fascículo 16. Instituto de Ecología A.C., Pátzcuaro.

Glass C. 1998. Guía Para la Identificación de Cactáceas Amenazadas de México. Vol. I. Comisión Nacional para el Conocimiento y Uso de la Biodiversidad y Cante, México, D.F.

Guzmán U., Arias S. y Dávila P. 2003. Catálogo de las Cactáceas Mexicanas. Universidad Nacional Autónoma de México y Comisión Nacional Para el Conocimiento y Uso de la Biodiversidad. México, D.F.

Hernández M. y Sánchez M. 2002. Informe de una nueva localidad de Mammillaria mathildae y una propuesta para modificar su categoría legal de conservación. Cactáceas y Suculentas Mexicanas 47:4-10.

Hobbs R.J. y Norton D.A. 1996. Towards a conceptual framework for restoration ecology. Restoration Ecology 4:93:110.

Holmes P.M. y Richardson D.M. 1999. Protocols for restoration based on recruitment dynamics, community structure and ecosystem function: perspectives from South African fynbos. Restoration Ecology 7:215-230.

International Plant Names Index. 2004. $<$ http://www.ipni.org >

Lot-Helgueras A. y Chiang F. Comps. 1986. Manual de Herbario. Administración y Manejo de Colecciones, Técnicas de Recolección y Preparación de Ejemplares Botánicos. Consejo Nacional de la Flora de México, A.C., México, D.F.

Lot-Helgueras A., Novelo-R. A., Olvera-G. M. y Ramírez-García P. 1999. Catálogo de Angiospermas Acuáticas de México. Instituto de Biología, Universidad Nacional Autónoma de México, México, D.F.

Mc Vaugh R. 1987. Leguminosae. En: Anderson W.R. Ed. Flora Novo-Galiciana, pp. 786, The University of Michigan Press, Ann Arbor, Michigan.

Mickel J.T. y Beitel J.M. 1988. Pteridophyte Flora of Oaxaca, México. Memoirs of the New York Botanical Garden 46:1-568.

Palmer M.A., Ambrose R.F. y Poff N.L. 1997. Ecological theory and community restoration ecology. Restoration Ecology 5:291-300.

Riba R. 1998. Pteridofitas mexicanas: Distribución y endemismo. En: Ramamoorthy T.P., Bye R., Lot A. y Fa J.E. (comps.). Diversidad Biológica de México. Orígenes y Distribución, pp. 369-384. Instituto de Biología, Universidad Nacional Autónoma de México, México, D.F.

Rzedowski J. 1978. Vegetación de México. Limusa, México, D.F.

Rzedowski J. 1991a. Diversidad y Orígenes de la flora fanerogámica de México. Acta Botanica Mexicana 14:3-22.

Rzedowski J. 1991b. El endemismo de la flora fanerogámica mexicana: Una apreciación analítica preliminar. Acta Botanica Mexicana 15:47-64.

Rzedowski J. y Guevara-Féfer F. 1992. Familia Burseraceae. Flora del Bajío y de Regiones Adyacentes. Fascículo 3. Instituto de Ecología, A.C., Pátzcuaro.

SEMARNAT [Secretaría del Medio Ambiente y Recursos 


\section{ANÁLISIS Florístico DE LA CAÑADA, QUERÉTARO}

Naturales]. 2001. Norma Oficial Mexicana NOM-059-ECOL2001, Protección ambiental-Especies nativas de México de flora y fauna silvestres-Categorías de riesgo y especificaciones para su inclusión, exclusión o cambio-Lista de especies en riesgo. Diario Oficial de la Federación. 2 a Sección, 6 de marzo de 2002.

Villaseñor J.L. 2003. Diversidad y distribución de las Magnoliophyta de México. Interciencia 28:160-167.

Villaseñor J.L. 2004. Los géneros de plantas vasculares de la flora de México. Boletín de la Sociedad Botánica de México 75:105135.

Fecha de recepción: 22 de abril de 2005

Versión corregida: 13 de octubre de 2005

Aceptado: 13 de octubre de 2005
$\mathrm{W}^{3}$ Tropicos. 2005. Missouri Botanical Garden's VAST nomenclatural database and associated authority files. $<\mathrm{http} / / /$ mobot.mobot.org/W3T/Search/vast.html>

Walter S. y Gillett H. Eds. 1998. 1997 IUCN Red List of Threatened Plants. Compilado por el World Conservation Monitoring Centre. IUCN-The World Conservation Union, Gland, Suiza y Cambridge, Reino Unido.

Zamudio S., Rzedowski J., Carranza E. y Calderón de R.G. 1992. La Vegetación del Estado de Querétaro. Instituto de Ecología, A.C., Pátzcuaro. 
Apéndice 1. Catálogo florístico de La Cañada, Querétaro. O = origen de la especie, FC = forma de crecimiento, D = distribución de la especie, E = Estatus de conservación de acuerdo con la NOM-059-ECOL-2001; 1 = especie nativa, 2 = originaria de Asia, 3 = Euroasiática, 4 = Caribeña, $5=$ Mediterráneo, $6=$ Europa, $7=$ África, $8=$ América tropical, $9=$ Sudamérica, $10=$ Norteamérica; $\mathrm{AB}=$ árbol, $\mathrm{AR}=$ arbusto, $\mathrm{BU}=$ bulbosa, $\mathrm{CR}=$ crasicaule, $\mathrm{EE}=$ enraizada emergente, $\mathrm{EP}=$ epífita, $\mathrm{HA}=$ hierba anual, $\mathrm{HP}=$ hierba perenne, $\mathrm{LF}=$ libre flotadora, $\mathrm{PA}=$ parásita, $\mathrm{RI}=$ rizomatosa, $\mathrm{RA}=$ rastrera, $\mathrm{RO}=$ rosetiforme, $\mathrm{TR}=$ trepadora o enredadera. $\mathrm{AM}=$ ampliamente distribuida en América, $\mathrm{CO}=$ cosmopolita, $\mathrm{D}=$ disyunta en América, $\mathrm{M}=\mathrm{México}, \mathrm{M} 1$ = Estados Unidos y México (Mega México 1), M2 = México y Centroamérica (Mega México 2), M3 = Estados Unidos, México y Centroamérica (Mega México 3), Q = Querétaro.

El guión significa sin estatus de conservación, $\mathrm{P}=$ peligro de extinción, $\mathrm{A}=$ amenazada, $\mathrm{R}=$ rara, $\mathrm{Pr}=$ protección especial. $\mathrm{El}$ asterisco indica registros previos que no fueron recolectados en el presente trabajo [para estas especies se incluye colector(es), número de colecta y el (los) herbario(s)].

\begin{tabular}{|c|c|c|c|c|}
\hline Grupo/ Familia / Género/ Especie & $\mathbf{O}$ & FC & D & $\mathbf{E}$ \\
\hline \multicolumn{5}{|l|}{ Bryophyta } \\
\hline \multicolumn{5}{|l|}{ Bryaceae } \\
\hline Bryum argenteum Hedw. & 1 & $\mathrm{HA}$ & AM & - \\
\hline \multicolumn{5}{|l|}{ Marchantiaceae } \\
\hline Marchantia sp. & 1 & $\mathrm{HA}$ & AM & - \\
\hline \multicolumn{5}{|l|}{ Pottiaceae } \\
\hline Didymodon vinealis (Brid.) R.H.Zander & 1 & $\mathrm{HA}$ & $\mathrm{CO}$ & - \\
\hline Pseudocrossidium replicatum (Taylor) R.H.Zander & 1 & $\mathrm{HA}$ & AM & - \\
\hline Tortula inermis (Brid.) Mont. & 1 & $\mathrm{HA}$ & $\mathrm{CO}$ & - \\
\hline Tortula obtusissima (Müll.Hal.) Mitt & 1 & $\mathrm{HA}$ & AM & - \\
\hline Weissia sp. & 1 & $\mathrm{HA}$ & $\mathrm{CO}$ & - \\
\hline \multicolumn{5}{|l|}{ PTERIDOPHYTA } \\
\hline \multicolumn{5}{|l|}{ Adiantaceae } \\
\hline Pellaea ovata (Desv.) Weath & 1 & RI & AM & - \\
\hline \multicolumn{5}{|l|}{ Polypodiaceae } \\
\hline Polypodium thyssanolepis A.Braun ex. Klotzsch & 1 & RI & AM & - \\
\hline \multicolumn{5}{|l|}{ Pteridaceae } \\
\hline Cheilanthes bonariensis (Willd.) Proctor & 1 & RI & AM & - \\
\hline Cheilanthes hookeri Domin & 1 & $\mathrm{RI}$ & AM & - \\
\hline Cheilanthes microphylla (Sw.) Sw. & 1 & RI & AM & - \\
\hline Cheilanthes myriophylla Desv. & 1 & RI & AM & - \\
\hline Cheilanthes sinuata (Lag. ex Sw.) Domin & 1 & RI & AM & - \\
\hline Cheilanthes sp. 1 & 1 & RI & AM & - \\
\hline Cheilanthes sp. 2 & 1 & RI & AM & - \\
\hline \multicolumn{5}{|l|}{ Selaginellaceae } \\
\hline Selaginella lepidophylla (Hook. et Grev.) Spring & 1 & $\mathrm{HP}$ & AM & - \\
\hline Selaginella rupincola Underw. & 1 & $\mathrm{HA}$ & AM & - \\
\hline \multicolumn{5}{|l|}{$\begin{array}{c}\text { ANTHOPHYTA } \\
\text { MAGNOLIOPSIDA }\end{array}$} \\
\hline \multicolumn{5}{|l|}{ Acanthaceae } \\
\hline Anisacanthus pumilus Nees & 1 & $\mathrm{AR}$ & M1 & - \\
\hline Anisacanthus quadrifidus (Vahl) Nees var. quadrifidus & 1 & $\mathrm{AR}$ & M1 & - \\
\hline * Carlowrightia parviflora (Buckley) Wassh. (E. Argüelles 1248, MEXU) & 1 & $\mathrm{HP}$ & M1 & - \\
\hline Dicliptera peduncularis Nees & 1 & $\mathrm{HP}$ & M1 & - \\
\hline * Justicia candicans (Ness) L.D.Benson et R.A.Darrow E. (E. Argüelles 2661, IEB) & 1 & $\mathrm{HP}$ & M1 & - \\
\hline Justicia caudata A.Gray & 1 & $\mathrm{HP}$ & M3 & - \\
\hline Ruellia lactea Cav. & 1 & $\mathrm{HA}$ & M2 & - \\
\hline Tetramerium nervosum Nees & 1 & RA & AM & - \\
\hline \multicolumn{5}{|l|}{ Amaranthaceae } \\
\hline Alternanthera pungens Humb. & 1 & RA & AM & - \\
\hline Gomphrena serrata L. & 1 & $\mathrm{HA}$ & AM & - \\
\hline Iresine calea (Ibáñez) Standl. & 1 & $\mathrm{AR}$ & AM & - \\
\hline Iresine cassiniiformis S.Schauer & 1 & $\mathrm{AR}$ & M2 & - \\
\hline Iresine schaffneri S.Watson & 1 & $\mathrm{AR}$ & M3 & - \\
\hline
\end{tabular}




\begin{tabular}{|c|c|c|c|c|}
\hline Grupo/ Familia / Género/ Especie & $\mathbf{O}$ & FC & D & $\mathbf{E}$ \\
\hline \multicolumn{5}{|l|}{ Anacardiaceae } \\
\hline Schinus molle L. & 9 & $\mathrm{AB}$ & AM & - \\
\hline \multicolumn{5}{|l|}{ Apiaceae } \\
\hline Arracacia sp. & 1 & $\mathrm{HP}$ & AM & - \\
\hline Apium leptophyllum (Pers.) F.Muell. ex Benth. & 8 & $\mathrm{HA}$ & AM & - \\
\hline \multicolumn{5}{|l|}{ Apocynaceae } \\
\hline Thevetia thevetioides (Kunth) K.Schum. & 1 & $\mathrm{AB}$ & M & - \\
\hline \multicolumn{5}{|l|}{ Asclepiadaceae } \\
\hline Asclepias linaria Cav. & 1 & AR & M1 & - \\
\hline Matelea pilosa (Benth.) Woodson & 1 & TR & M & - \\
\hline Metastelma angustifolium Turcz. & 1 & TR & M & - \\
\hline Sarcostemma pannosum Decne. & 1 & TR & M1 & - \\
\hline \multicolumn{5}{|l|}{ Asteraceae } \\
\hline Acourtia alamanii (DC.) Reveal et R.M.King & 1 & $\mathrm{HP}$ & M & - \\
\hline Acourtia platyphylla (A.Gray) Reveal et R.M.King & 1 & $\mathrm{HP}$ & M & - \\
\hline Ageratum corymbosum Zucc. & 1 & $\mathrm{HP}$ & AM & - \\
\hline Ambrosia confertiflora DC. & 1 & $\mathrm{HP}$ & AM & - \\
\hline Aster subulatus Michx. & 10 & $\mathrm{HA}$ & AM & - \\
\hline Baccharis pteronioides DC. & 1 & $\mathrm{HP}$ & AM & - \\
\hline Baccharis salicifolia (Ruiz et Pav.) Pers. & 9 & AR & AM & - \\
\hline Bidens ferulifolia (Jacq.) DC. & 8 & $\mathrm{HA}$ & $\mathrm{CO}$ & - \\
\hline Bidens odorata Cav. & 4 & $\mathrm{HA}$ & $\mathrm{CO}$ & - \\
\hline Brickellia rapunculoides (DC.) McVaugh & 1 & $\mathrm{HP}$ & M1 & - \\
\hline Brickellia veronicifolia (Kunth) A.Gray & 1 & $\mathrm{HP}$ & M1 & - \\
\hline Dahlia coccinea Cav. & 1 & $\mathrm{HP}$ & M3 & - \\
\hline Dyssodia tenuifolia (Cass) Loes. & 1 & $\mathrm{HA}$ & M1 & - \\
\hline Eupatorium sp. & 1 & $\mathrm{HP}$ & AM & - \\
\hline Erigeron sp. & 1 & $\mathrm{HP}$ & AM & - \\
\hline Florestina pedata (Cav.) Cass. & 1 & $\mathrm{HA}$ & M2 & - \\
\hline Gnaphalium luteoalbum L. & 2 & $\mathrm{HA}$ & $\mathrm{CO}$ & - \\
\hline Gnaphalium oxyphyllum DC. & 1 & $\mathrm{HA}$ & AM & - \\
\hline Helenium mexicanum Kunth & 1 & $\mathrm{HA}$ & AM & - \\
\hline Helianthus aff. laciniatus A.Gray & 10 & $\mathrm{HP}$ & AM & - \\
\hline Heterotheca inuloides var. rosei B.Wagenkn. & 1 & $\mathrm{HP}$ & M1 & - \\
\hline Montanoa tomentosa Cerv. & 1 & $\mathrm{HP}$ & M1 & - \\
\hline Piqueria trinervia Cav. & 1 & $\mathrm{HP}$ & AM & - \\
\hline * Pectis prostrata Cav. (E. Argüelles 2379, IEB, MEXU) & 1 & $\mathrm{HA}$ & AM & - \\
\hline Pluchea odorata (L.) Cass. & 1 & $\mathrm{HP}$ & AM & - \\
\hline * Porophyllum macrocephalum DC. (E. Argüelles 2377, MEXU) & 1 & $\mathrm{HA}$ & AM & - \\
\hline Sabazia humilis (Kunth) Cass. & 1 & $\mathrm{HA}$ & M1 & - \\
\hline Senecio salignus DC. & 1 & AR & AM & - \\
\hline Trixis inula Crantz & 1 & AR & AM & - \\
\hline * Trixis mexicana Lex. (E. Argüelles 673, ENCB, MEXU) & 1 & AR & M & - \\
\hline Verbesina serrata Cav. & 1 & $\mathrm{AR}$ & AM & - \\
\hline Viguiera linearis (Cav.) Sch.Bip. ex Hemsl. & 1 & $\mathrm{HP}$ & AM & - \\
\hline Zinnia peruviana $\mathrm{L}$. & 9 & $\mathrm{HA}$ & AM & - \\
\hline \multicolumn{5}{|l|}{ Basellaceae } \\
\hline Anredera ramosa (Moq.) Eliasson & 1 & TR & AM & - \\
\hline \multicolumn{5}{|l|}{ Bignoniaceae } \\
\hline Tecoma stans (L.) Juss. ex Kunth & 1 & AR & AM & - \\
\hline \multicolumn{5}{|l|}{ Brassicaceae } \\
\hline Descurainia virletii E.Fourn. & 1 & $\mathrm{HA}$ & AM & - \\
\hline Eruca sativa Mill. & 3 & $\mathrm{HA}$ & $\mathrm{CO}$ & - \\
\hline Halimolobos berlandieri O.E.Schulz & 1 & $\mathrm{HP}$ & M & - \\
\hline Lepidium virginicum L. & 10 & $\mathrm{HA}$ & $\mathrm{CO}$ & - \\
\hline \multicolumn{5}{|l|}{ Bombacaceae } \\
\hline Ceiba aesculifolia (Kunth) Britten et Baker $\mathrm{f}$. & 1 & $\mathrm{AB}$ & AM & - \\
\hline
\end{tabular}




\begin{tabular}{|c|c|c|c|c|}
\hline Grupo/ Familia / Género/ Especie & $\mathbf{O}$ & FC & $\mathbf{D}$ & $\mathbf{E}$ \\
\hline \multicolumn{5}{|l|}{ Boraginaceae } \\
\hline Heliotropium foliosissimum J.F.Macbr. & 1 & RA & M2 & - \\
\hline Lithospermum distichum Ortega & 1 & $\mathrm{HP}$ & $\mathrm{AM}$ & - \\
\hline \multicolumn{5}{|l|}{ Buddlejaceae } \\
\hline Buddleja parviflora Kunth & 1 & $\mathrm{AB}$ & M1 & - \\
\hline \multicolumn{5}{|l|}{ Burseraceae } \\
\hline Bursera fagaroides (Kunth) Engl. & 1 & $\mathrm{AB}$ & M1 & - \\
\hline Bursera palmeri S.Watson & 1 & $A B$ & M1 & - \\
\hline \multicolumn{5}{|l|}{ Cactaceae } \\
\hline Cylindropuntia imbricata (Haw.) F.M.Knuth subsp. imbricada & 1 & $\mathrm{CR}$ & M1 & - \\
\hline Coryphantha erecta (Lem.) Lem. & 1 & $\mathrm{CR}$ & M & - \\
\hline Coryphantha radians (DC.) Britton et Rose & 1 & $\mathrm{CR}$ & M & $\mathrm{R}$ \\
\hline Echinocactus platyacanthus Link et Otto & 1 & $\mathrm{CR}$ & M & $\operatorname{Pr}$ \\
\hline Echinocereus cinerascens (DC.) Lem. subsp. cinerascens & 1 & $\mathrm{CR}$ & M & - \\
\hline Ferocactus echide (DC.) Britton et Rose & 1 & $\mathrm{CR}$ & M & - \\
\hline Ferocactus latispinus (Haw.) Britton et Rose & 1 & $\mathrm{CR}$ & M & - \\
\hline Isolatocereus dumortieri (Scheidw.) Backeb. & 1 & $\mathrm{CR}$ & M & - \\
\hline Mammillaria magnimamma Haw. & 1 & $\mathrm{CR}$ & M & - \\
\hline Mammillaria mathildae Kraehenb. et Krainz & 1 & $\mathrm{CR}$ & Q & $\mathrm{P}$ \\
\hline Mammillaria polythele Mart. subsp. polythele & 1 & $\mathrm{CR}$ & M & - \\
\hline Marginatocereus marginatus (DC.) Backeb. & 1 & $\mathrm{CR}$ & M & - \\
\hline Myrtillocactus geometrizans (Mart. ex Pfeiff.) Console var. geometrizans & 1 & $\mathrm{CR}$ & M & - \\
\hline Nopalea cochenillifera (L.) Salm-Dyck & 8 & $\mathrm{CR}$ & AM & - \\
\hline Opuntia joconostle F.A.C.Weber & 1 & $\mathrm{CR}$ & M & - \\
\hline Opuntia megacantha Salm-Dyck & 1 & $\mathrm{CR}$ & M & - \\
\hline Opuntia pubescens J.C.Wendl. ex Pfeiff. & 1 & $\mathrm{CR}$ & $\mathrm{AM}$ & - \\
\hline Opuntia robusta J.C.Wendl. & 1 & $\mathrm{CR}$ & M & - \\
\hline Opuntia streptacantha Lem. & 1 & $\mathrm{CR}$ & M & - \\
\hline Opuntia tomentosa Salm-Dyck & 1 & $\mathrm{CR}$ & M & - \\
\hline Peniocereus serpentinus (Lag. et Rodr.) N.P.Taylor & 1 & $\mathrm{CR}$ & M & - \\
\hline Pereskiopsis diguetii (F.A.C.Weber) Britton et Rose & 1 & $\mathrm{CR}$ & M & - \\
\hline $\begin{array}{l}\text { Stenocactus dichroacanthus (Mart. ex Pfeiff.) A.Berger } \\
\text { ex Backeb. et F.M.Knuth }\end{array}$ & 1 & $\mathrm{CR}$ & M & - \\
\hline Stenocereus queretaroensis (F.A.C.Weber) Buxb. & 1 & $\mathrm{CR}$ & M & - \\
\hline \multicolumn{5}{|l|}{ Capparaceae } \\
\hline Polanisia uniglandulosa (Cav.) DC. & 1 & $\mathrm{HA}$ & M1 & - \\
\hline \multicolumn{5}{|l|}{ Convolvulaceae } \\
\hline Dichondra argentea Humb. et Bonpl. ex Willd. & 1 & RA & AM & - \\
\hline Evolvulus alsinoides (L.) L. & 1 & RA & $\mathrm{CO}$ & - \\
\hline Ipomoea cristulata Hallier f. & 1 & TR & M1 & - \\
\hline Ipomoea murucoides Roem. et Schult. & 1 & $\mathrm{AB}$ & M2 & - \\
\hline Ipomoea pubescens Lam. & 1 & TR & AM & - \\
\hline Ipomoea purpurea (L.) Roth & 8 & TR & AM & - \\
\hline Ipomoea sp. & 1 & TR & AM & - \\
\hline \multicolumn{5}{|l|}{ Crassulaceae } \\
\hline Echeveria coccinea (Cav.) DC. & 1 & $\mathrm{CR}$ & M & - \\
\hline Echeveria mucronata Schltdl. & 1 & $\mathrm{CR}$ & M1 & - \\
\hline Pachyphytum compactum Rose & 1 & $\mathrm{CR}$ & M & - \\
\hline Sedum dendroideum Moc. et Sessé ex DC. & 1 & $\mathrm{CR}$ & M & - \\
\hline Sedum ebracteatum Moc. et Sessé ex DC. & 1 & $\mathrm{CR}$ & M & - \\
\hline \multicolumn{5}{|l|}{ Cucurbitaceae } \\
\hline Cucurbita foetidissima Kunth & 10 & RA & $\mathrm{AM}$ & - \\
\hline Cucurbita radicans Naudin & 1 & RA & M & - \\
\hline Cyclanthera dissecta (Torr. et A.Gray) Arn. & 10 & $\mathrm{TR}$ & M3 & - \\
\hline \multicolumn{5}{|l|}{ Dipsacaceae } \\
\hline Dipsacus sp. & 5 & $\mathrm{HP}$ & $\mathrm{CO}$ & - \\
\hline
\end{tabular}




\begin{tabular}{|c|c|c|c|c|}
\hline Grupo/ Familia / Género/ Especie & $\mathbf{O}$ & FC & D & $\mathbf{E}$ \\
\hline \multicolumn{5}{|l|}{ Euphorbiaceae } \\
\hline Acalypha subviscida S.Watson & 1 & $\mathrm{HP}$ & M2 & - \\
\hline Croton ciliatoglanduliferus Ortega & 1 & AR & M3 & - \\
\hline Euphorbia dentata Michx. & 10 & RA & AM & - \\
\hline Euphorbia hirta var. procumbens (DC.) N.E.Br. & 8 & $\mathrm{RA}$ & AM & - \\
\hline Euphorbia prostrata Aiton & 8 & RA & $\mathrm{AM}$ & - \\
\hline Euphorbia sp. & 1 & $\mathrm{HA}$ & AM & - \\
\hline Jatropha dioica Cerv. & 1 & CR & M1 & - \\
\hline Manihot mexicana I.M.Johnst. & 1 & $\mathrm{HP}$ & AM & - \\
\hline Ricinus communis $\mathrm{L}$. & 7 & AR & $\mathrm{CO}$ & - \\
\hline \multicolumn{5}{|l|}{ Fabaceae } \\
\hline Acacia angustissima (Mill.) Kuntze & 1 & AR & AM & - \\
\hline Acacia farnesiana (L.) Willd. & 1 & AR & $\mathrm{AM}$ & - \\
\hline Acacia pennatula (Schltdl. et Cham.) Benth. & 1 & AR & AM & - \\
\hline Calliandra capillata Benth. & 1 & AR & M & - \\
\hline Calliandra eriophylla Benth. & 1 & AR & M1 & - \\
\hline Dalea bicolor Humb. et Bonpl. ex Willd. & 1 & AR & M1 & - \\
\hline Dalea versicolor Zucc. & 1 & AR & M3 & - \\
\hline Erythrina coralloides DC. & 1 & $\mathrm{AB}$ & M & A \\
\hline Eysenthardtia polystachya (Ortega) Sarg. & 1 & $\mathrm{AB}$ & M1 & - \\
\hline Galactia brachystachys Benth. & 1 & TR & M1 & - \\
\hline Lysiloma microphylla Benth. & 1 & $\mathrm{AB}$ & AM & - \\
\hline Mimosa aculeaticarpa Ortega & 1 & AR & AM & - \\
\hline Mimosa lacerata Rose & 1 & AR & M3 & - \\
\hline Prosopis laevigata (Humb. et Bonpl. ex Willd.) M.C.Johnst. & 1 & $\mathrm{AB}$ & M1 & - \\
\hline Phaseolus atropurpureus Sessé et Moc. ex DC. & 1 & TR & AM & - \\
\hline Senna polyantha (Moc. et Sessé ex Collad.) H.S.Irwin et Barneby & 1 & $\mathrm{AB}$ & M & - \\
\hline \multicolumn{5}{|l|}{ Hydrophyllaceae } \\
\hline Nama origanifolium Kunth & 1 & $\mathrm{HP}$ & M1 & - \\
\hline \multicolumn{5}{|l|}{ Krameriaceae } \\
\hline Krameria pauciflora Moc. et Sessé ex DC. & 1 & RA & M1 & - \\
\hline \multicolumn{5}{|l|}{ Lamiaceae } \\
\hline Hyptis albida Kunth & 1 & $\mathrm{HP}$ & AM & - \\
\hline Salvia hirsuta Jacq. & 1 & $\mathrm{HA}$ & M1 & - \\
\hline Salvia melissodora Lag. & 1 & $\mathrm{HP}$ & M1 & - \\
\hline Salvia reptans Jacq. & 1 & $\mathrm{HP}$ & M3 & - \\
\hline Salvia tiliifolia Vahl & 1 & $\mathrm{HA}$ & AM & - \\
\hline Stachys coccinea Ortega & 1 & $\mathrm{HA}$ & $\mathrm{AM}$ & - \\
\hline Teucrium cubense Jacq. & 1 & $\mathrm{HA}$ & AM & - \\
\hline \multicolumn{5}{|l|}{ Loasaceae } \\
\hline Eucnide lobata (Hook.) A.Gray & 1 & $\mathrm{HP}$ & AM & - \\
\hline * Mentzelia aspera L. (E. Argüelles 1283, MEXU) & 1 & $\mathrm{HA}$ & AM & - \\
\hline Mentzelia hispida Willd. & 1 & $\mathrm{HP}$ & M3 & - \\
\hline \multicolumn{5}{|l|}{ Loranthaceae } \\
\hline Psittacanthus schiedeanus (Schltdl. et Cham.) Blume & 1 & PA & AM & - \\
\hline Psittacanthus palmeri (S.Watson) Barlow et Wiens & 1 & $\mathrm{PA}$ & AM & - \\
\hline \multicolumn{5}{|l|}{ Lytrhaceae } \\
\hline *Cuphea wrightii A.Gray var. wrightii (E. Argüelles 2660, 3010, IEB) & 1 & $\mathrm{HA}$ & AM & - \\
\hline Heimia salicifolia Link & 1 & AR & AM & - \\
\hline \multicolumn{5}{|l|}{ Malpighiaceae } \\
\hline Gaudichaudia cynanchoides Kunth & 1 & TR & $\mathrm{AM}$ & - \\
\hline \multicolumn{5}{|l|}{ Malvaceae } \\
\hline * Allowissadula floribunda (Schltdl.) Fryxell (E. Argüelles 2732, IEB, QMEX) & 1 & AR & M & - \\
\hline * Anoda maculata Fryxell (E. Argüelles 1434, ENCB, MEXU, QMEX) & 1 & $\mathrm{HP}$ & M & - \\
\hline Herissantia crispa (L.) Brizicky & 9 & RA & AM & - \\
\hline Malva parviflora L. & 3 & $\mathrm{HA}$ & $\mathrm{CO}$ & - \\
\hline
\end{tabular}




\begin{tabular}{|c|c|c|c|c|}
\hline Grupo/ Familia / Género/ Especie & $\mathbf{O}$ & FC & D & $\mathbf{E}$ \\
\hline Malvastrum coromandelianum (L.) Garcke & 9 & $\mathrm{HA}$ & AM & - \\
\hline Sphaeralcea angustifolia (Cav.) G.Don & 8 & $\mathrm{HA}$ & AM & - \\
\hline \multicolumn{5}{|l|}{ Martyniaceae } \\
\hline Proboscidea louisiana (Mill.) Wooton et Standl. & 10 & $\mathrm{HA}$ & AM & - \\
\hline \multicolumn{5}{|l|}{ Molluginaceae } \\
\hline * Mollugo verticillata L. (E. Argüelles 2074, MEXU) & 8 & $\mathrm{HA}$ & AM & - \\
\hline \multicolumn{5}{|l|}{ Nyctaginaceae } \\
\hline * Boerhavia gracillima Heimerl (E. Argüelles 1291, 3235, IEB, MEXU) & 1 & $\mathrm{HP}$ & M1 & - \\
\hline Mirabilis jalapa L. & 1 & $\mathrm{HP}$ & AM & - \\
\hline Mirabilis longiflora L. & 1 & $\mathrm{HP}$ & M3 & - \\
\hline Oxybaphus glabrifolius (Ortega) Vahl & 8 & $\mathrm{HA}$ & AM & - \\
\hline Pisoniella arborescens (Lang. et Rodr.) Standl. & 1 & AR & AM & - \\
\hline \multicolumn{5}{|l|}{ Oleaceae } \\
\hline Forestiera angustifolia Torr. & 1 & AR & M1 & - \\
\hline Menodora helianthemoides Bonpl. & 1 & $\mathrm{HP}$ & M1 & - \\
\hline \multicolumn{5}{|l|}{ Onagraceae } \\
\hline Gaura coccinea ex Pursh & 1 & RA & AM & - \\
\hline Lopezia racemosa Cav. & 1 & $\mathrm{HA}$ & AM & - \\
\hline Oenothera rosea L'Hér. ex Aiton & 1 & $\mathrm{HA}$ & AM & - \\
\hline \multicolumn{5}{|l|}{ Oxalidaceae } \\
\hline Oxalis decaphylla Kunth & 10 & BU & M1 & - \\
\hline Oxalis latifolia Kunth & 1 & $\mathrm{BU}$ & AM & - \\
\hline \multicolumn{5}{|l|}{ Papaveraceae } \\
\hline Argemone ochroleuca Sweet subsp. ochroleuca & 1 & $\mathrm{HA}$ & AM & - \\
\hline \multicolumn{5}{|l|}{ Phytolaccaceae } \\
\hline Rivina humilis L. & 1 & $\mathrm{HA}$ & AM & - \\
\hline \multicolumn{5}{|l|}{ Plantaginaceae } \\
\hline Plantago major L. & 3 & $\mathrm{HA}$ & $\mathrm{CO}$ & - \\
\hline \multicolumn{5}{|l|}{ Plumbaginaceae } \\
\hline Plumbago pulchella Boiss. & 1 & $\mathrm{HP}$ & M1 & - \\
\hline \multicolumn{5}{|l|}{ Polemoniaceae } \\
\hline Loeselia coerulea (Cav.) G.Don & 1 & RA & M1 & - \\
\hline Loeselia mexicana (Lam.) Brand & 1 & $A R$ & M1 & - \\
\hline \multicolumn{5}{|l|}{ Polygalaceae } \\
\hline Polygala barbeyana Chodat & 1 & $\mathrm{HP}$ & M1 & - \\
\hline \multicolumn{5}{|l|}{ Polygonaceae } \\
\hline Polygonum mexicanum Small & 1 & $\mathrm{EE}$ & M1 & - \\
\hline Rumex crispus $\mathrm{L}$. & 3 & $\mathrm{HA}$ & $\mathrm{CO}$ & - \\
\hline \multicolumn{5}{|l|}{ Portulacaceae } \\
\hline Portulaca oleracea L. & 3 & RA & $\mathrm{CO}$ & - \\
\hline Portulaca pilosa L. & 1 & RA & AM & - \\
\hline Talinopsis frutescens A.Gray. & 1 & AR & M1 & - \\
\hline Talinum paniculatum (Jacq.) Gaertn. & 1 & $\mathrm{HP}$ & AM & - \\
\hline \multicolumn{5}{|l|}{ Ranunculaceae } \\
\hline Clematis pitcheri Torr. et A.Gray & 1 & TR & M1 & - \\
\hline \multicolumn{5}{|l|}{ Rhamnaceae } \\
\hline Colubrina triflora Brongn. ex Sweet & 1 & $\mathrm{AR}$ & M2 & - \\
\hline Condalia velutina I.M.Johnst. & 1 & AR & M & - \\
\hline Karwinskia humboldtiana (Willd. ex Roem. et Schult.) Zucc. & 1 & AR & AM & - \\
\hline \multicolumn{5}{|l|}{ Rubiaceae } \\
\hline Bouvardia multiflora (Cav.) Schult. et Schult.f. & 1 & AR & AM & - \\
\hline Bouvardia ternifolia (Cav.) Schltdl. & 1 & AR & M1 & - \\
\hline \multicolumn{5}{|l|}{ Rutaceae } \\
\hline Casimiroa edulis La Llave et Lex. & 1 & $A B$ & AM & - \\
\hline Salicaceae & & & & \\
\hline Salix bonplandiana Kunth & 1 & $A B$ & AM & - \\
\hline
\end{tabular}




\begin{tabular}{|c|c|c|c|c|}
\hline Grupo/ Familia / Género/ Especie & $\mathbf{O}$ & FC & D & $\mathbf{E}$ \\
\hline \multicolumn{5}{|l|}{ Sapindaceae } \\
\hline Cardiospermum halicacabum L. & 1 & TR & $\mathrm{CO}$ & - \\
\hline \multicolumn{5}{|l|}{ Scrophulariaceae } \\
\hline Castilleja sp. & 1 & AR & AM & - \\
\hline Maurandya antirrhiniflora Humb. et Bonpl. ex Willd. & 1 & $\mathrm{TR}$ & AM & - \\
\hline \multicolumn{5}{|l|}{ Solanaceae } \\
\hline Datura stramonium L. & 1 & $\mathrm{HA}$ & AM & - \\
\hline Nicotiana glauca Graham & 9 & $A R$ & AM & - \\
\hline Petunia parviflora Juss. & 9 & RA & DI & - \\
\hline Physalis cinerascens (Dunal) Hitchc. & 1 & $\mathrm{HA}$ & AM & - \\
\hline Solanum cardiophyllum Lindl. & 1 & $\mathrm{HA}$ & M3 & - \\
\hline Solanum elaeagnifolium Cav. & 9 & $\mathrm{HA}$ & AM & - \\
\hline Solanum nigrescens M.Martens et Galeotii & 1 & $\mathrm{HA}$ & AM & - \\
\hline Solanum rostratum Dunal & 1 & $\mathrm{HA}$ & AM & - \\
\hline \multicolumn{5}{|l|}{ Sterculiaceae } \\
\hline Ayenia aff. jaliscana S.Watson & 1 & $\mathrm{HP}$ & M & - \\
\hline \multicolumn{5}{|l|}{ Ulmaceae } \\
\hline Celtis pallida Torr. & 1 & AR & DI & - \\
\hline \multicolumn{5}{|l|}{ Verbenaceae } \\
\hline Aloysia gratissima (Gillies et Hook.) Tronc. & 1 & AR & DI & - \\
\hline Bouchea prismatica (L.) Kuntze & 8 & $\mathrm{HA}$ & AM & - \\
\hline Lantana camara L. & 1 & $A R$ & AM & - \\
\hline Lantana hirta Graham & 1 & $\mathrm{HP}$ & AM & - \\
\hline Glandularia elegans (Kunth) Umber & 1 & $\mathrm{HP}$ & M & - \\
\hline * Priva mexicana (L.) Pers. (E. Argüelles 3012, 3175, IEB, MEXU) & 1 & $\mathrm{HP}$ & M2 & - \\
\hline Verbena menthiefolia Benth. & 1 & $\mathrm{HP}$ & M3 & - \\
\hline \multicolumn{5}{|l|}{ Viscaceae } \\
\hline Phoradendron brachystachyum (DC.) Nutt. & 1 & PA & AM & - \\
\hline \multicolumn{5}{|l|}{ Vitaceae } \\
\hline Cissus sicyoides L. & 1 & TR & AM & - \\
\hline \multicolumn{5}{|l|}{ Zygophyllaceae } \\
\hline * Kallstroemia rosei Rydb. (E. Argüelles 3232, IEB) & 1 & $\mathrm{HA}$ & M & - \\
\hline \multicolumn{5}{|l|}{ LILIOPSIDA } \\
\hline \multicolumn{5}{|l|}{ Agavaceae } \\
\hline Agave salmiana Otto ex Salm-Dick & 1 & $\mathrm{RO}$ & M3 & - \\
\hline Yucca filifera Chabaud & 1 & $\mathrm{RO}$ & M3 & - \\
\hline \multicolumn{5}{|l|}{ Alliaceae } \\
\hline Milla biflora Cav. & 1 & BU & M3 & - \\
\hline \multicolumn{5}{|l|}{ Asphodelaceae } \\
\hline Aloe vera (L.) Burm.f. & 7 & $\mathrm{RO}$ & $\mathrm{CO}$ & - \\
\hline \multicolumn{5}{|l|}{ Amaryllidaceae } \\
\hline * Zephyranthes carinata Herb. (E. Argüelles 351, MEXU) & 1 & BU & M & - \\
\hline Zephyranthes fosteri Traub & 1 & BU & M & - \\
\hline \multicolumn{5}{|l|}{ Bromeliaceae } \\
\hline Hechtia glomerata Zucc. & 1 & $\mathrm{RO}$ & M3 & - \\
\hline Tillandsia lepidosepala L.B.Sm. & 1 & $\mathrm{RO}$ & M3 & - \\
\hline Tillandsia recurvata (L.) L. & 1 & $\mathrm{EP}$ & AM & - \\
\hline Tillandsia sp. & 1 & $\mathrm{RO}$ & AM & - \\
\hline \multicolumn{5}{|l|}{ Commelinaceae } \\
\hline Commelina diffusa Burm.f. & 2 & $\mathrm{HA}$ & $\mathrm{AM}$ & - \\
\hline Tradescantia crassifolia Cav. & 1 & $\mathrm{HP}$ & $\mathrm{M} 2$ & - \\
\hline \multicolumn{5}{|l|}{ Cyperaceae } \\
\hline Cyperus spectabilis Link & 1 & $\mathrm{EE}$ & $\mathrm{AM}$ & - \\
\hline \multicolumn{5}{|l|}{ Lemnaceae } \\
\hline Lemna gibba L. & 1 & LF & $\mathrm{CO}$ & - \\
\hline
\end{tabular}


José Alejandro CABrera-Luna y Maricela GómeZ-SÁncheZ

\begin{tabular}{|c|c|c|c|c|}
\hline Grupo/ Familia / Género/ Especie & $\mathbf{O}$ & FC & D & $\mathbf{E}$ \\
\hline \multicolumn{5}{|l|}{ Orchidaceae } \\
\hline Laelia aff. speciosa (Kunth) Schltr. & 1 & $\mathrm{EP}$ & M & A \\
\hline \multicolumn{5}{|l|}{ Poaceae } \\
\hline Aristida adscensionis L. & 9 & $\mathrm{HA}$ & $\mathrm{CO}$ & - \\
\hline Aristida divaricata Humb. et Bonpl. ex Willd. & 1 & $\mathrm{HP}$ & $\mathrm{AM}$ & - \\
\hline Bothriochloa barbinodis (Lag.) Herter & 1 & $\mathrm{HP}$ & $\mathrm{AM}$ & - \\
\hline Bouteloua curtipendula (Michx.) Torr. & 1 & $\mathrm{HP}$ & $\mathrm{AM}$ & - \\
\hline Bouteloua hirsuta Lag. & 10 & $\mathrm{HP}$ & $\mathrm{AM}$ & - \\
\hline Bouteloua repens (Kunth) Scribn. & 1 & $\mathrm{HP}$ & $\mathrm{AM}$ & - \\
\hline Buchloë dactyloides (Nutt.) Engelm. & 1 & $\mathrm{HP}$ & AM & - \\
\hline Eleusine multiflora Hochst. ex A.Rich. & 7 & $\mathrm{HA}$ & $\mathrm{CO}$ & - \\
\hline Enneapogon desvauxii P.Beauv. & 1 & $\mathrm{HP}$ & AM & - \\
\hline Eragrostis cilianensis (All.) Vignolo ex Janch. & 6 & $\mathrm{HA}$ & $\mathrm{CO}$ & - \\
\hline Leptochloa aff. aquatica Scribn. et Merr. & 1 & $\mathrm{HP}$ & M & - \\
\hline Leptochloa sp. & 1 & $\mathrm{HP}$ & $\mathrm{AM}$ & - \\
\hline Muhlenbergia implicata (Kunth) Trin. & 1 & $\mathrm{HA}$ & $\mathrm{AM}$ & - \\
\hline Panicum fasciculatum Sw. & 8 & $\mathrm{HA}$ & $\mathrm{AM}$ & - \\
\hline Panicum sp. & 1 & $\mathrm{HP}$ & $\mathrm{AM}$ & - \\
\hline Melinis repens (Will.) C.E.Hubb. & 7 & $\mathrm{HP}$ & $\mathrm{CO}$ & - \\
\hline Setaria geniculata P.Beauv. & 1 & $\mathrm{HP}$ & $\mathrm{CO}$ & - \\
\hline \multicolumn{5}{|l|}{ Typhaceae } \\
\hline Typha domingensis Pers. & 1 & $\mathrm{EE}$ & $\mathrm{CO}$ & - \\
\hline
\end{tabular}

